Mots. Les langages du politique

\title{
Symbolique et portée politiques du geste de Rabia en Égypte
}

The gesture Rabia in Egypt. Symbolic values and political impact

Simbólica y dimensión política del gesto de Rabia en Egipto

Belkacem Benzenine

\section{OpenEdition}

Journals

Édition électronique

URL : https://journals.openedition.org/mots/22255

DOI : $10.4000 /$ mots. 22255

ISSN : 1960-6001

Éditeur

ENS Éditions

Édition imprimée

Date de publication : 9 mai 2016

Pagination : $99-115$

ISBN : 978-2-84788-793-8

ISSN : 0243-6450

\section{Référence électronique}

Belkacem Benzenine, "Symbolique et portée politiques du geste de Rabia en Égypte », Mots. Les

langages du politique [En ligne], 110 | 2016, mis en ligne le 09 mai 2018, consulté le 22 avril 2022. URL : http://journals.openedition.org/mots/22255 ; DOl : https://doi.org/10.4000/mots.22255

\section{(C) ENS Éditions}




\section{Symbolique et portée politiques du geste de Rabia en Égypte}

L'un des signes qui ont le plus marqué la période «post-printemps arabe » est celui des quatre doigts de la main, utilisé par les Frères musulmans d’Égypte après la décision du gouvernement de mettre fin à leurs rassemblements sur les places publiques Nahda et Rabia Adawiyya en août 2013. Ces quatre doigts levés symbolisent en effet, d'un côté, la résistance et le défi des Frères musulmans, et de l'autre, la «martyrisation » de ceux des leurs tombés lors des affrontements avec les forces de l'ordre. Ce geste, repris dans le logo ci-dessous, occupe une place importante dans l'espace public, non seulement en Égypte mais aussi dans d'autres pays arabes et musulmans.

Les quatre doigts de Rabia', qui renvoient au nom d'une place du Caire, représentent, depuis les événements de 2013, l'un des gestes politiques les plus controversés dans le monde arabe et musulman. Sur les réseaux sociaux, R4bia est un signe abondamment commenté par les internautes. On le trouve dans les domaines sportif et artistique ; il est utilisé dans presque tous les «événements » politiques; il est porteur de plusieurs expressions politiques, mais selon les Frères musulmans, qui se le sont approprié, il renvoie à leurs principes et à leurs idéaux.

Nous nous proposons d'apporter un éclairage sur les divers sens accordés à ce geste et à ses usages en Égypte, dans quelques autres pays arabes et en Turquie, où il a essaimé. En quoi ce signe est-il caractéristique de l'opposition islamiste? Dans le contexte du «printemps arabe», quelle est sa portée politique et idéologique? Comment s’inscrit-il dans le conflit entre les Frères musulmans et les autorités égyptiennes? Notre attention se portera également sur les campagnes menées par les médias et les acteurs politiques égyptiens contre ce geste, pour montrer comment, autour de lui, s'affrontent les islamistes et leurs opposants. Au-delà de la conjoncture d'émergence du geste, nous nous intéresserons particulièrement à sa portée politique et sociale pour tenter de comprendre en quoi il traduit les visées des Frères musulmans, l'un des mouvements politiques les plus influents dans le monde arabe.

1. Souvent écrit en caractères latins: R4BIA (rabia signifiant « quatre mains » ou « quatrième » en arabe). 


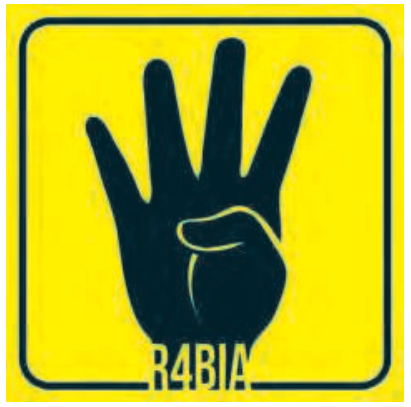

Logo reproduisant le "geste de Rabia » Source: Wikimedia Commons.

Notre étude repose sur un corpus ouvert constitué de photographies, d'articles de presse, de messages sur les réseaux sociaux et de différents écrits attachés à cette période décisive de l'histoire de l'Égypte post-printemps arabe $^{2}$. Après avoir rappelé l'importance des lieux de mobilisation, en 2011 comme en 2013, nous envisagerons la diffusion du geste puis les différents sens qui ont pu lui être attribués, pour finir par sa transformation en enjeu symbolique dans le conflit politique en Égypte.

\section{Les précédents : Maydan Tahrir comme symbole de protestation politique}

Les événements déclenchés dans plusieurs pays arabes à partir de la fin de 2010 ne cessent d'attirer l'attention des chercheurs sur leurs dimensions politiques, sociales, culturelles et économiques. Ces événements sont marqués, comme c'est le cas de plusieurs révoltes et révolutions, par des rapports conflictuels entre le peuple et l'État. Ils concernent également des lieux et espaces emblématiques qui constituent une référence, voire forgent leur identité même. Maydan3 Tahrir, au Caire, fut ainsi et demeure l'une des places publiques les plus «politiques» dans les pays arabes. Tahrir, qui signifie «libération», a fini par symboliser une volonté de changement, d'émancipation, d'unité des citoyens, et à certains égards le courage et le défi du peuple ou de certaines catégories du peuple dans l'expression de leurs revendications politiques et sociales. "Le Maydan» est devenu aussi en peu de temps «l'espace public» de rassemblement, beaucoup d’Égyptiens étant convaincus que tout se joue

2. Nous avons recueilli de nombreuses photographies du geste de Rabia effectué lors des manifestations organisées en Égypte et dans d'autres pays; les articles relatifs aux événements et à ce geste émanent de la presse arabe et sont parus dès le début des manifestations des Frères musulmans. Nous avons également examiné d'autres textes provenant de la communication de la confrérie, des partis politiques et du gouvernement égyptien.

3. Maydan signifie «place» en arabe. 
sur cette place transformée en dix-huit jours seulement en épicentre symbolique et physique de la révolution égyptienne (El Chazli, 2012 ; Rushdy, 2011).

Les slogans scandés y exprimaient, dès le 25 janvier 2011, la volonté de chasser le pouvoir autoritaire incarné par le président Moubarak, sa famille et l'oligarchie qui le soutenait. Bien que ce but ait été atteint, les Égyptiens n'ont pas fait tomber le régime. En effet, si la «révolution» du 25 janvier a engendré un discours de changement et de liberté, elle a été «relativement neutre» à certains égards, au moins dans ses débuts, puisqu'elle ne dégageait pas d'orientation idéologique claire. Les manifestants, tout en représentant différentes tendances, n'étaient alors regroupés sous aucune bannière politique et ne montraient pas de signes partisans distinctifs très marqués. Comme si seul «le Maydan», qui concrétisait les symboles et les gestes du changement révolutionnaire, remplissait un vide que ni les islamistes, ni les forces politiques et religieuses, ni Moubarak et son régime n'étaient capables de combler. Les slogans de la révolution étaient pleins de colère et d'amertume, mais en même temps d'émotion et d'espoir. Le mot d'ordre «Irhal» («Dégage!»)4 résumait à lui seul «la volonté du Maydan», consacrée volonté populaire. L'autorité et la « légalité » portées par le lieu ont été traduites et renforcées par plusieurs épisodes : c'est en ce lieu, en effet, que le pouvoir égyptien est allé le 3 février 2011 chercher les jeunes pour dialoguer avec le nouveau vice-président Omar Suleiman. C'est là que les chefs militaires, dont le maréchal Tantawi, dirigeant du Conseil suprême des forces armées en février 2011, sont venus assurer le peuple de la neutralité de l'institution militaire. C'est là encore qu'Essam Charaf, désigné au poste de Premier ministre, s'est présenté devant les manifestants, le 4 mars 2011, pour prêter serment, considérant qu'il tirait sa légitimité du peuple.

Les manifestants de Maydan Tahrir exprimaient les aspirations d'une incoercible révolution, joignant les gestes à la parole : les mains levées vers le ciel, sur la tête, en mouvement, ils témoignaient de la volonté du peuple de chasser Moubarak du pouvoir. Serrant les poings l'un sur l'autre en signe d'unité, ils scandaient : «Le peuple et l'armée forment une seule main ». Significativement, la gestualité de la révolution de 2011, au commencement du moins, affichait le défi et appelait à l'union. Les poings fermés et levés des mains droites et les signes des deux doigts en $\mathrm{V}$ pour souligner respectivement le combat et la victoire symbolisaient une action collective inédite pour les mouvements de protestation politique dans les pays arabes. Des mains ouvertes étaient exhibées, sur les doigts desquelles étaient écrits des slogans comme «dégage», " liberté », « dignité». Elles étaient aussi levées, portant les drapeaux d’Égypte, signe fort de patriotisme, ou bien elles brandissaient des cartouches vides utilisées par les forces de l'ordre contre les manifestants, afin de protester contre la violence. Toutefois, aucun geste particulier ne traduisait alors des tendances

4. Sur le slogan d'origine tunisienne: Azouzi, 2013. 
politiques et idéologiques. Même si une orientation «de gauche» tendait à rayonner, cela ne tenait qu'à la nature sociale des revendications (emploi, justice sociale, dignité, égalité, etc.).

Comment qualifier alors les événements plus récents, ceux qui ont secoué le Caire entre juin et août 2013, concentrés sur les deux grandes places Nahda et Rabia Adawiyya ?Émeute, insurrection, rébellion, révolte, sédition ou soulèvement? C'est cette fois la destitution du président Mohamed Morsi que réclament les manifestants, et les événements de Rabia Adawiyya et de Nahda vont ouvrir une autre ère politique, marquée par la fin du « règne » des Frères musulmans et leur entrée en guerre contre le nouveau pouvoir mis en place par le général Abdel Fattah al-Sissi.

\section{Rabia : au commencement était le lieu}

Les confrontations entre les anti et les pro-Morsi débutent le 30 juin 2013, jour anniversaire de son arrivée au pouvoir, après que celui-ci a pris des décisions jugées non constitutionnelles et contraires à l'esprit de la «révolution de janvier ». Les grands rassemblements organisés par l'opposition, représentée par différentes tendances, marquent la vie politique de l'Égypte post-printemps arabe. Les opposants se regroupent massivement à Maydan Tahrir. Cette place est de nouveau le symbole de la protestation politique. Le mouvement Tamarrud («rébellion »), soutenu par certaines forces politiques de la gauche et des libéraux, mène une campagne acharnée visant la destitution du président. Alors que ce groupement envisage d'organiser un rassemblement massif le 30 juin 2013, exigeant l'organisation d'une élection présidentielle anticipée, les soutiens de Morsi commencent à développer un slogan de «sauvegarde de la légitimité» (shari ‘iyya). Tamarrud, mené par de jeunes dirigeants, est considéré comme l'expression d'un clivage générationnel (Barbary, Adib Doss, 2014, p. 157). Ses membres espèrent réaliser une nouvelle «vague de la révolution égyptienne», tout en jouant un rôle important sur le plan international, pour donner à leur action une légitimité populaire (Alianak, 2014, p. 93 et s.)

L'institution militaire, incarnée par le ministre de la Défense et chef des forces armées, le général Sissi, soutenu par des leaders religieux - représentant alAzhar et l'Église copte - et politiques de différentes tendances, y compris le parti salafiste Nour, annonce la fin de pouvoir du président Morsi, le gel provisoire de la Constitution et l'organisation de l'élection présidentielle. Les militaires et l'appareil judiciaire apparaissent alors comme les vainqueurs (Arjomand, 2015, p. 38). Pour la première fois dans le monde arabe post-révolutions de 2010-2011, un président élu est destitué et la Constitution gelée (Messiha, Teulon, 2013, p.139).

Les places Nahda et Rabia Adawiyya deviennent dès lors les bastions de la résistance des Frères musulmans (représentant leur confrérie historique, son 
aile politique, le parti Liberté et Justice et leurs alliés islamistes). Les manifestants y affichent leur attachement à la légitimité présidentielle et leur volonté de poursuivre la mobilisation jusqu'au retour du président déchu. La première place, qui tire son nom de la statue qui l'orne, Nahdat Masr ("Renaissance de l'Égypte »), est située dans le quartier historique de Gizeh, non loin des représentations diplomatiques et de l'université du Caire. La seconde place, plus grande, est la plus emblématique : Rabia Adawiyya est le siège des rassemblements les plus importants des Frères musulmans. C'est là qu'ils organisent leurs démonstrations de force et que se retrouvent généralement leurs chefs. Cette place est stratégique, certes, parce qu'elle permet de contrôler l'accès à plusieurs grandes rues du Caire, mais cette raison n'est pas la seule à justifier le choix de la localisation : elle abrite en effet une grande mosquée éponyme. Construite en 1993, c'est l'une des plus célèbres de la ville. Une association, « Rabia Adawiyya pour le développement et l'entraide sociale », y mène une très présente action de bienfaisance, contrôlant les annexes de la mosquée : hôpital, centre de dialyse, salle de réception dans les occasions funèbres, centres d'informatique, d'études en développement humain et d'études islamiques5. La mosquée devient un lieu de logistique et la place sert d'abord d'espace de démonstration de force et de point d'appui au mouvement de protestation des islamistes, avant de devenir le refuge des chefs des Frères musulmans recherchés par la justice.

Mais outre ces caractéristiques stratégiques et religieuses, la place que les islamistes ont retenue pour se regrouper et soutenir le président Morsi porte un nom évocateur : «Rabia Adawiyya » n'est pas seulement un toponyme mais constitue, dans l'histoire islamique, un symbole de patience et d'adoration divine. Née à Bassora en 717, Rabia est la quatrième fille de sa très modeste famille. "Sainte par excellence de l'hagiographie sunnite», selon Massignon (1987, p. 6), son personnage, souvent mythifié, célébré par un film égyptien (Rabi'a al-'Adawiyya, 1963, Niazi Mustafa) et de nombreux travaux académiques, est une allégorie de l'idée d'épreuve et de persévérance pour l'amour de Dieu. Certes, les Frères musulmans, qui n'avaient pas imaginé la tournure des événements, n'avaient pas vu d'emblée, dans le nom du Maydan Rabia, l'attribut de la souffrance : c'est surtout durant les affrontements avec les forces de l'ordre, précédés d'une forte campagne médiatique menée contre l'organisation islamiste, qu'ils ont commencé à effectuer un rapprochement entre le nom de Rabia Adawiyya et la souffrance «pour l'amour de Dieu». On remarquera que l'idée de souffrance est utilisée dans ce contexte à mauvais escient par les Frères musulmans quand ils font référence à la vie de la «mystique». En effet, on ne retrouve pas dans ses biographies (Smith, 2010; Benghal, 2000) ce qui ferait d'elle une martyre de la vérité. Néanmoins, il semble que la référence à la

5. Cette association a été mise sous contrôle du gouvernement égyptien, comme tous les biens appartenant à l'association des Frères musulmans. 
souffrance soit mise en avant pour renvoyer au mystique islamique dans certains cas, comme la maladie, parce qu'elle est « conçue comme une grâce [que le mystique musulman] accueille comme une douleur qui lui est envoyée pour éprouver sa foi » (Carcenac, 2013). Aussi, comme c'est le cas dans la tradition musulmane, la souffrance pourrait être présentée, puisqu'il est question de Rabia Adawiyya, comme étant une «volonté du Seigneur» et par suite « une bénédiction » (Annestay, 2009, p. 86). Force est de constater ainsi le paradoxe qui consiste à adopter un symbole gestuel identique à celui du soufisme, alors que dans la littérature des Frères musulmans, le soufisme est une forme de religiosité qui est sinon rejetée, du moins considérée comme opposée à leur idéologie ${ }^{6}$.

L'évacuation des places Rabia et Nahda, exigée par le gouvernement, laisse des centaines de morts et de blessés. Pour les Frères musulmans, cette issue tragique représente un acte patriotique et un «empressement vers Dieu» (Massignon, 1987, p.6). Sur la place, citations coraniques et chants religieux résonnent en un mélodieux concert qui sera utilisé par les animateurs des rassemblements pour attirer la sympathie des musulmans du monde entier. Les paroles de la sainte sont chantées, des vidéos diffusées largement sur les réseaux sociaux. Certaines chaînes satellitaires, dont Al-Jazeera, retransmettent en direct les événements de la place Rabia. C'est à la fin des rassemblements, qui vont durer du 28 juin au 14 août 2013, que certains sympathisants du président Morsi commencent à afficher le fameux geste devant les caméras pour souligner leur présence sur les lieux. Il reste difficile d'indiquer avec certitude quand et où ce geste fut utilisé pour la première fois, même si l'on trouve sur les réseaux sociaux, cité comme pionnier sans que cela puisse être attesté, le nom d'un jeune handicapé sympathisant des Frères musulmans, décédé lors des émeutes. Si l'on note la présence de ce geste à la fin des événements de la place Rabia, c'est plutôt sa large diffusion comme symbole - non pas des Frères musulmans, mais du refus de la destitution du président Morsi (et donc du déni de légitimité du nouveau pouvoir) - qui donne à la période suivante une impulsion décisive.

Présente depuis la fin des années vingt dans la vie politique, sociale et religieuse égyptienne, l'association des Frères musulmans n'avait connu qu'un logo, dont seules les couleurs avaient légèrement évolué avec le temps, outre quelques modifications graphiques. Ce logo, un cercle renfermant deux sabres entrecroisés surmontés d'un Coran ainsi qu'un mot unique, «Préparez »7 , accompagné parfois du slogan de l'organisation, «l'Islam est la solu-

6. Et ce même si Hassan al-Banna, le fondateur des Frères musulmans, considérait son mouvement comme étant «salafiste, une réalité soufie». Notons aussi que le régime égyptien, sous le règne de Nasser notamment, utilisait les confréries soufies contre les Frères musulmans pour les isoler de la vie politique et sociale. Voir à ce sujet Luizard, 1990.

7. Premier mot d'un verset coranique : «Préparez, pour lutter contre eux [les infidèles], tout ce que vous trouverez de force et de cavaleries » (Coran, 8, 60). Hassan al-Banna lui-même définit son association comme étant religion, État, nation et sabre. Voir Mitchell, 1963, p. 233. 
tion ${ }^{8}$, résume une partie de l'idéologie du groupe, qui vise à fonder un État islamique reposant sur les principes coraniques. Or, à Maydan Rabia, le logo des Frères n'est guère affiché. On y voit plutôt des portraits du président déchu et le drapeau de l'Égypte, ainsi que des banderoles reflétant les revendications des manifestants. Souhaitant montrer qu'il s'agit d'une affaire qui ne les concerne pas seuls, la confrérie préfère organiser les rassemblements au nom d'un «Front national de défense de la légitimité » qui regroupe les principales forces islamiques opposées à la destitution de Morsi. Elle peut ainsi jouer sur ses réseaux clientélaires, qui l'ont aidée à récolter des voix à l'occasion des différents scrutins (Aclimandos, 2011).

Les manifestations des Frères musulmans rassemblés pour soutenir Morsi à l'été 2013 auront en tout cas donné une symbolique gestuelle à l'action et à la protestation islamistes. Les événements des deux Maydan (Rabia et Nahda) ont en ce sens été instrumentalisés par la confrérie afin de se présenter comme défenseur de la légitimité et, ipso facto, le «souffre-douleur» d'un «coup d’État» renversant un président «démocratiquement» élu. Relevons toutefois le conflit entre les notions de "légalité» (shari'iyya) et de «légitimité» (mashru'iyya). Car si, pour les partisans de Morsi, il est surtout question de respecter la loi - et donc la légalité découlant de son élection démocratique -, leurs opposants invoquent l'illégitimité de son pouvoir : à leurs yeux, Morsi a trahi l'esprit de la révolution et enfreint la Constitution en soumettant son interprétation à l'idéologie des Frères.

Dans l'histoire du monde musulman, peu de gestes ont produit autant de polémiques que celui de Rabia. Sans doute, en premier lieu, parce que ce dernier a connu une diffusion inattendue.

\section{La diffusion du geste de protestation islamiste}

Très rapidement après les violentes confrontations entre les Frères musulmans et les forces de l'ordre, le geste est affiché de manière systématique dans les manifestations organisées en Égypte, puis dans d'autres pays comme la Turquie, la Tunisie et la Jordanie, pour dénoncer la répression et afficher en même temps un soutien à l'ancien président. Ainsi, lever les quatre doigts de la main prend une signification de plus en plus politique, symbolisant la poursuite du combat de la confrérie. Et le geste va perdurer malgré la tension accrue de la situation politique.

Dans ce contexte, un nom célèbre est lié au geste de Rabia, celui de Recep Tayyip Erdogan, le président turc. Exprimant plus que sa solidarité avec les Frères musulmans, avec qui il partage certains idéaux, Erdogan, en levant ses

8. Les Frères musulmans se sont toujours présentés comme «la seule force de l'opposition » et «la seule réponse au système» (Carré, Michaud, 1983). 
quatre doigts en l'air trois jours après l'intervention des forces de l'ordre à Maydan Rabia, donne au geste sa dimension internationale. Car pour lui, intervenant lors d'une réunion du Parti de la justice et du développement (AKP), ce geste n'est pas seulement « un symbole de la cause juste du peuple égyptien, il est devenu une façon de dire non à l'injustice dans le monde entier ». En dressant ses doigts devant les cadres de son organisation et les journalistes, Erdogan annonce qu'il importe de s'opposer aux coups d'État et de soutenir les justes causes de l'humanité9. Il est ainsi le premier chef politique étranger à faire du geste de Rabia le symbole de la lutte pour la justice et pour le respect de la volonté populaire. Et il répète son geste à l'envi, ostensiblement, accordant aux Frères musulmans un soutien fort. Rabia prend alors un sens spécifique, renvoyant au respect de la légitimité et aux pratiques démocratiques. Avec Erdogan, le geste est aussi utilisé pour dire «stop ». La manière dont les Frères musulmans le réalisent est d'ailleurs souvent celle d'un geste d'arrêt.

Connus par leur capacité de mobilisation et d'organisation, due à plus de huit décennies d'action politique, la plupart du temps dans la clandestinité, les Frères musulmans ont saisi cette opportunité pour donner au geste une extension internationale, au-delà du lieu du massacre. Il est désormais utilisé pour afficher sa solidarité avec leur cause. Pour ce faire, leurs adeptes vont reproduire le signe en l'affichant sur les murs, les banderoles, les étiquettes et les autocollants.

À l'occasion du procès du président Morsi et des chefs des Frères musulmans en novembre 2013, le geste de Rabia est montré avec une exaltation mêlée d'opiniâtreté. Souriant et sacrifiant au nouveau geste ritualisé devant les caméras, les prisonniers affichent leur attachement au lieu symbolique de Maydan Rabia tout en rappelant qu'ils sont des victimes politiques d'un régime "oppresseur». Lors de plusieurs manifestations sportives, des joueurs égyptiens profitent de la couverture médiatique pour arborer le geste. Souvent effectué en direct, et dans des compétitions internationales organisées en Égypte comme ailleurs, cet acte est considéré comme une provocation et une subversion par les autorités égyptiennes - nous y reviendrons.

Avec le temps, les positions se radicalisent dans les deux camps, «signe d'une suspension de l'autolimitation, l'une des propriétés des conjonctures fluides» (Boutaleb, 2014, p. 482). La crise s'étend, sa dimension internationale s'accentue et le geste de Rabia tend à provoquer des réactions de plus en plus vives. Il est montré non seulement à l'occasion de manifestations en Égypte, mais aussi en Tunisie, où les partisans du mouvement al-Nahda affichent ainsi leur soutien aux Frères musulmans ${ }^{10}$. De façon imprévue et surprenante, il est égale-

9. Comme le montrent plusieurs vidéos diffusées sur les réseaux sociaux. Voir également https:// www.youtube.com/watch?v=6Qmo7BKldc 8 (dernière consultation des sites mentionnés dans cet article : 20 janvier 2016).

10. Plus récemment, le président tunisien Moncef Marzouki n’a pas hésité à faire le geste de Rabia lors d'une rencontre organisée le 6 mai 2015 par les Frères musulmans à Paris, ce qui a été 
ment utilisé plusieurs fois à l'occasion du pèlerinage de 2013 à La Mecque, dont les images sont diffusées en direct et vues par des millions de téléspectateurs, qui suivent cet événement religieux des plus importants pour l'islam. On voit, sur le mont Arafat, des pèlerins utiliser des parapluies ornés des quatre doigts. Sur les réseaux sociaux, certains diffusent des selfies montrant le geste, ou portant de petites pancartes qui rappellent les événements de Maydan Rabia. De tels comportements, bien que limités, ont été planifiés, les Frères ayant lancé des appels aux pèlerins du monde entier afin qu'ils saisissent l'occasion de ce grand rassemblement religieux pour défendre leur «cause » en faisant le fameux geste. Cela malgré les avertissements des autorités saoudiennes, hostiles à la politisation du pèlerinage. Les milieux médiatiques et politiques ont d'ailleurs acueilli le geste avec colère, en Arabie Saoudite comme en Égypte.

Mais la campagne la plus vive et ardente pour défendre le geste de Rabia et le promouvoir, notamment sur la scène internationale, est celle menée sur Internet. Un site web, r4bia.com, est créé fin 2013 afin de soutenir «les principes de Rabia », avec pour slogan : «Nous n'oublions pas». Ce site, consacré à la propagande et à la défense de la position des Frères musulmans, victimisés, présente le geste comme un symbole de la liberté. Sur certains autres sites, des t-shirts et des casquettes où il figure sont mis en vente. Une plate-forme internationale du nom de «Rabia» déclare même le 14 août 2014 "journée mondiale Rabia » pour commémorer les événements de l'année précédente ${ }^{11}$. C'est surtout grâce aux réseaux sociaux que le geste gagne en popularité. Sur Twitter et Facebook en particulier, les internautes se mobilisent pour en faire un signe d'appartenance idéologique et politique. Certains, parmi eux, l'adoptent comme image personnelle, d'autres publient leurs selfies en faisant le geste. L'action politique et la sécurité étant devenues difficiles et précaires en Égypte pour les Frères musulmans, ceux-ci ouvrent fin 2013 une chaîne de télévision à Istanbul, qu'ils baptisent Rabia et dotent d'un logo de couleur jaune représentant les quatre doigts.

Mais que peut signifier le geste pour ceux qui le font? Examinons d'abord une première série d’interprétations données au geste et au logo qui le reprend.

\section{Une pluralité de lectures du geste}

Lever les quatre doigts pouvait d'abord apparaître, à l'origine, comme une manière d'afficher une distinction gestuelle par rapport aux manifestants

interprété comme « un signe de ralliement et de soutien inconditionnel à la cause de la confrérie». Voir : http://blogs.mediapart.fr/blog/salah-horchani/100515/la-photo-du-jour-ca-y-estmoncef-marzouki-introduit-la-main-de-rabaa-comme-signe-du-salut-collec.

11. Voir http://www.dailysabah.com/mideast/2014/07/09/rabia-platform-declares-august-14world-rabia-day. 
rassemblés place Tahrir pour réclamer le départ de Morsi, qui utilisaient les deux doigts en signe de victoire. Ces deux doigts en $V$ représentant un signe universel, utilisé en particulier aux États-Unis, ne pouvaient évidemment convenir aux Frères musulmans qui, en outre, passaient de la victoire (ils contrôlaient presque toutes les institutions importantes du pays : présidence, gouvernement, parlement) à la sortie du pouvoir. Pourtant, aussi paradoxal que cela puisse paraître, les quatre doigts ont pu être considérés parfois comme signifiant une double victoire (il s'agirait, selon certaines lectures ${ }^{12}$, d'un double signe de V : la première victoire contre le régime de Moubarak, la seconde, celle des Frères musulmans à l'occasion des élections législatives et présidentielle, ainsi qu'au référendum constitutionnel). D'autres interprètes estiment que le geste de Rabia est principalement porteur d'un message adressé à la nation arabe, puisqu'il ne peut être appréhendé que par rapport à la langue arabe et au contexte propre aux Arabes (journal Al-Ghad). Pour d'autres encore, les quatre doigts font essentiellement référence à la durée du mandat du président Morsi, constitutionnellement élu pour quatre ans. Rabia, qui renvoie, nous l'avons vu, au chiffre quatre en arabe, peut aussi correspondre au fait que Morsi est le quatrième président d’Égypte (après Nasser, Sadate et Moubarak13).

Les commentaires se succèdent surtout chez les Frères musulmans pour conférer au geste une interprétation religieuse crédible et séductrice. Sont alors évoqués les quatre mois sacrés (hurum) durant lesquels la paix doit être respectée, les quatre premiers califes de l'islam, les quatre imams (dans la tradition sunnite du droit islamique), pour expliquer que l'usage de ce geste n'est pas anodin et qu'il puise dans l'imaginaire islamique commun ${ }^{14}$ sa force symbolique. Pour donner au signe et au geste leur dimension internationale et attirer la sympathie et la solidarité des musulmans partout dans le monde, plusieurs acceptions sont proposées. Les Frères musulmans font de leur nouveau signe de ralliement une référence aux valeurs de liberté et de paix. Par Rabia, ils renvoient non seulement au «massacre du Maydan Rabia » et à la destitution de Morsi, mais aussi aux thèmes qui constituent leur idéologie, comme la fin du sionisme et du capitalisme, la libération de la Palestine, les «martyrs» et la solidarité musulmane.

La distinction des différentes composantes du logo permet également de mettre en évidence le travail de symbolisation mené par les partisans de la

12. Voir par exemple http://www.theatlantic.com/international/archive/2013/o9/what-thishand-gesture-means-for-egypts-future/279730/ et http://arabi21.com/story/741955/ethttp:// arabi21.com/story/741955/.

13. En réalité, il est le cinquième président puisque après la révolution du 23 juillet 1952, Mohamed Naguib est rapidement écarté par Nasser. Son bref passage au pouvoir explique sans doute qu'il ne soit pas souvent cité parmi les présidents égyptiens.

14. Nous empruntons à Mohamed Arkoun l'expression d' "imaginaire islamique commun » par laquelle il désigne «l'ensemble des croyances données à percevoir, à penser, à vivre comme vraies et qui n'admettent aucune intervention de la raison critique indépendante : celle-ci devient source d'hérésie, de dévoiement, si elle n'accepte pas d'être exclusivement la servante de l'imaginaire » (Arkoun, 1986, p.13). 
cause soutenue. Très rapidement, ce geste de mémoire et de défi a été doté d'un symbole pictographique représentant les quatre doigts de la main droite 15 (voir le logo en introduction). Simple et expressive, facile à reconnaître et à saisir, la figure prend une forme unique et colorée. Pour trouver toute sa place dans les champs politique et médiatique, elle est dessinée avec la position finale des doigts en noir sur fond jaune. Elle apparaît sous cette forme presque officielle sur de grandes banderoles à l'occasion de la première manifestation organisée à l'étranger pour soutenir le président Morsi et les victimes des violences de Maydan Rabia.

De la Turquie, qui se positionne comme premier pays de soutien aux Frères musulmans, on apprend que le signe a été dessiné par deux jeunes designers turcs. Dans une interview largement reprise par les médias égyptiens et arabes, Saliha Eren et Cihat Döleş déclarent être à l'origine du dessin. Tous deux travaillent pour le compte du site d'information Haber Seyret, la première comme journaliste et le second comme designer. Le geste est officiellement diffusé sur ce site avec un encadré noir et une graphie latine, $\mathrm{R}_{4} \mathrm{BI} \mathrm{A}^{16}$, qui apparaît en jaune sur le fond noir de la jonction de la paume et du poignet. Le geste prend forme de sigle. Il est diffusé sur le site personnel de Cihat Döleş ${ }^{17}$. Les deux jeunes Turcs donnent au geste sa valeur idéographique, très symbolique, qui acquiert plus de visibilité et d'intensité avec l'amplification de la mobilisation pour s'opposer au « coup d'État contre Morsi ». Ils s'expliquent en particulier sur le choix des couleurs : d'après eux, le geste est tout d'abord « un cri contre le massacre qui a eu lieu à Maydan Rabia ». Pour Saliha Eren, la couleur jaune proviendrait d'une image qu'elle a observée quelques années plus tôt : «Le soleil brillait sur le dôme du Rocher (Jérusalem). Je voyais cette image à chaque fois que les musulmans se rebellent contre la cruauté qu'ils exercent sur eux-mêmes ou sur les autres. » Selon la même interprétation du document et de ses couleurs,

le noir renvoie au rassemblement même de Rabia, où une femme habillée en noir priait en levant ses mains ouvertes au milieu de la place. Cela m'a fait penser à la sainte Kaaba que nous sacralisons, parce qu'elle représente pour nous [musulmans] la direction de la prière (kibla) qui est notre chemin à chaque fois que nous sommes égarés dans le monde, la Kaaba est notre boussole... (haberseyret.com, version arabe)

Le signe est ainsi fortement teinté de sacralité religieuse, référé à deux lieux très vénérés chez les musulmans : la Kaaba, elle-même considérée dans l'imaginaire islamique comme «la maison de Dieu», est pour eux un lieu chargé de

15. Les documents observés montrent que c'est le plus souvent la main droite qui est levée pour faire le geste de Rabia. La main gauche peut l'être aussi, comme l'attestent certaines photographies, mais plutôt lorsque les deux mains sont utilisées.

16. Le chiffre quatre correspond à la lettre A selon le dictionnaire anglophone des mots populaires Urban Dictionnary diffusé en ligne: http://www.urbandictionary.com/.

17. http://cihatdoles.com/tr/wp-content/uploads/r4bia.jpg. 
symboles. Petit édifice bâti, selon les historiens musulmans, par le prophète Abraham, elle est symbole de l'islam, de l'unité des croyants, de la genèse même de la religion musulmane et de deux obligations religieuses : la prière et le pèlerinage. Elle «représente le centre lui-même... elle est le symbole de toute position centrale dans l'islam... » (Austin et al., 1977, p. 66). La pierre dite «noire » constitue un élément de la Kaaba, collée sur l'un de ses murs. La Kaaba est couverte de brocart noir orné de caractères coraniques en or. Le rapport établi avec la Kaaba pour dessiner des doigts symbolisant la résistance et un «massacre » semble étrange. La journaliste turque cherche en réalité à islamiser le geste et à appeler à l'unité des musulmans pour faire face à l'«injustice » frappant les Frères. Quant au jaune, il renverrait aussi à un autre lieu hautement symbolique et sacré chez les musulmans : la mosquée de Jérusalem, qui constitue dans la tradition religieuse la première orientation de la prière. Le conflit arabo-israélien a fait de cette grande mosquée un lieu de conflit qui suscite une forte sympathie chez la communauté musulmane pratiquante. Comme la Kaaba, cette mosquée est souvent présente dans des tableaux qui décorent les maisons et les bureaux privés et publics.

Dans la tradition arabe et islamique, le jaune...

associé à l'or, au soleil, au feu est essentiellement la couleur de l'ambiguïté. Il est pâleur et richesse, luminosité et ternissure, profusion matérielle et pauvreté spirituelle. La symbolique du jaune dans la culture arabe rejoint ainsi celle de la culture occidentale dont Roger Bastide a analysé les subtils mécanismes tous orientés vers l'idée de trahison. La palette islamique admet bien le jaune, mais c'est avec beaucoup de circonspection et beaucoup de sentiments mêlés. (Bouhdiba, 1980, p. 69)

L’idée de «sentiments mêlés » semble trouver toute son actualité si l'on se réfère à l'évolution tragique des événements de Maydan Rabia. Toutefois, le choix du jaune, lié à la trahison, paraît équivoque, d'autant plus que les Frères musulmans présentent le geste de Rabia comme un symbole de fidélité au président Morsi. Peut-être veulent-ils ainsi marquer une nouvelle période politique donnant à leur «révolution » une couleur propre et dire aussi qu’ils sont euxmêmes victimes d'une trahison ${ }^{18}$ ?

L'explication du choix chromatique par Saliha Eren est largement dominée par une connotation religieuse "affective», qui puise dans l'imaginaire islamique et s'inscrit dans la temporalité du conflit arabo-israélien. Le geste de Rabia devient par excellence, sinon un signe d'appartenance aux Frères musulmans, du moins de sympathie avec eux, cette fois au nom de l'islam et de ses lieux saints.

18. Dans les manifestations des Frères musulmans, on voit parfois des manifestants faire le geste de Rabia tout en brandissant des photos de Sissi lors de sa désignation comme ministre de la Défense, prêtant serment devant le président Morsi, cela pour souligner la trahison et la prévarication. 
Cependant, pour les Frères musulmans, le signe peut aussi renvoyer à une lecture différente. En effet, selon leur organe de presse Al-Hurriyya wal-'Adâla (La Liberté et la Justice), « la couleur jaune suscite l'activité dans l'appareil neurologique puisqu'il renforce les cellules du cerveau ouvrant l'appétit pour la vie d'une manière générale, elle est réjouissante aux regards ». Référence est faite ici à une citation coranique rappelant un dialogue entre Moise et les Juifs sur la vache à sacrifier, de couleur jaune ${ }^{19}$. En s'appuyant sur de prétendues connaissances savantes ( $M$. Amir Saleh ${ }^{20}$, dont la notoriété ne repose en réalité que sur la médecine naturelle et traditionnelle), les Frères musulmans ne tentent-ils pas de justifier le choix du jaune par l'autorité de la «science », en conciliant ainsi des impératifs de communication et des prétentions religieuses? II n'est pas exclu non plus que les couleurs aient été plus ou moins inspirées par le logo d’Amnesty International, où le jaune et le noir font référence à une liberté qui brille par son éclat, d'autant que les Frères musulmans tentent de faire de leur lutte une affaire de liberté précisément, de droits de l'Homme et de dignité.

Quant à la main, elle possède dans l'imaginaire islamique une forte charge symbolique, renvoyant au don, à la prière et à l'invocation. Comme l'écrit M. Chebel, « la main s'émeut, refuse, exprime la crainte, le regret, ou l'avarice »:

[...] la main droite est positive, la main gauche est néfaste ${ }^{21}$. Il est dit dans le Coran : «celui qui recevra son livre [des comptes dans lequel se trouvent transcrites toutes les actions commises ici-bas] de la main gauche dira : malheur à moi... » LXI 25. En revanche, celui qui recevra son livre dans la main droite sera jugé avec mansuétude. La main est souvent mise en corrélation avec la souveraineté divine. (Chebel, 1993, p. 256)

Les quatre doigts de la main droite levée sont pour les Frères musulmans synonymes de serment donné et de foi.

On se doute que la diffusion du geste de Rabia, ainsi interprété, ne pouvait laisser sans réaction les autorités égyptiennes. Cela a donné lieu à des batailles de références qui ont fait de ce geste un véritable enjeu politique et médiatique.

\section{La bataille des interprétations et la riposte politique et médiatique}

La propagation large et rapide du geste de Rabia est bien sûr de nature à effrayer les médias publics et privés proches du nouveau régime, qui y voient le symbole

19. Selon Jacques Berque (1995), il s'agit d'une «scène mi-satirique » qui « fait ressortir les résistances des mauvais croyants, qui font jouer l'argutie, voire l'ironie, pour échapper au commandement de Dieu».

20. Voir http://www.amir-saleh.com. L'auteur considère que l'écriture noire sur fond jaune est stimulante pour la mémoire et les capacités intellectuelles, chez les étudiants notamment, et qu'elle exerce en outre un impact positif sur le tempérament et le comportement des individus...

21. C'est le cas aussi dans la religion chrétienne (Feuillet, 2004, p. 71). 
du refus de la nouvelle autorité et l'emblème d'une révolte islamiste. Il est certain que pour les Frères musulmans et les sympathisants de Morsi, il correspond à un défi adressé au nouveau régime, mais aussi à la résistance des islamistes réclamant un retour à la légitimité. La confrérie répondant 22 à une riposte très offensive menée par le gouvernement et les médias égyptiens qui lui est favorable, le geste de Rabia se transforme en véritable enjeu symbolique dans la confrontation politique. Né d'un rassemblement pour défendre la légitimité du président déchu à la suite d'affrontements sanglants, il prend alors tout son sens politique (Khalifan, 2015; Cole, 2014 ; Sabea, 2014).

Le nouveau régime du général Sissi mène de son côté une campagne de stigmatisation contre le geste de Rabia. Les médias qui le soutiennent s'engagent dans cette campagne d'une manière agressive pour délégitimer le geste. Celui-ci est montré comme un emblème de discorde, de rébellion et de division de la société égyptienne, qu'on cherche à présenter unie au côté de son dirigeant. Les Frères musulmans sont même accusés d'adopter le geste en référence à un symbole à quatre doigts de la littérature maçonnique, invraisemblable interprétation 23 amplement reprise sur les réseaux sociaux pour dénigrer les opposants et bannir leur gestuelle. Certains médias inventent même des similitudes gestuelles pour diffamer les Frères musulmans : le Moulin rouge est ainsi évoqué sur les réseaux sociaux pour prétendre que le signe est emprunté à une affiche du célèbre cabaret parisien. Cette information est bien sûr démentie par les Frères musulmans, qui la prennent pour une calomnie. Une dernière version, belliciste, est suggérée par certains medias quant au signe de R4bia tel qu'il est adopté sur les réseaux sociaux et dans certains écrits journalistiques : $\mathrm{R}=$ Ready $; 4$ = for (four) $; \mathrm{B}=$ Brotherhood, $\mathrm{I}=$ Independent, $\mathrm{A}=$ Army $^{24}$. Mais la création de cette armée présumée des terroristes n'a jamais été attestée...

Force est de constater qu'une propagande intensive et généralisée est menée, des deux côtés, autour d'un geste devenu plus que jamais significatif d'un conflit politique, indissociable d'une nouvelle époque dans la vie politique égyptienne, marquée par la volonté du pouvoir de mettre fin à l'existence de l'association des Frères musulmans.

Face à l'ampleur prise par «le mouvement de Rabia» sur les réseaux sociaux, le gouvernement s'est décidé à agir. Le ministre de l'Intérieur annonce ainsi, fin 2013, l'application de l'article 86 du code pénal qui stipule que « toute personne appartenant à ou soutenant ou diffusant les idées d'une organisa-

22. Un «Mouvement des jeunes contre le coup d’État» est créé pour soutenir la protestation des Frères musulmans. Usant d'un logo jaune montrant un jeune debout avec les deux mains levées, cette organisation adopte le même geste de Rabia dans ses manifestations de solidarité avec les victimes des affrontements. Un groupement analogue pour les étudiants est également constitué après les émeutes de Rabia, portant le même logo.

23. Il n'existe aucun geste franc-maçon comparable.

24. Voir: http://religionresearch.org/closer/2013/09/01/r4bia-the-symbolic-construction-of-protest/, consulté le 2 décembre 2015. 
tion terroriste sera punie par la loi ». L'association des Frères musulmans étant considérée comme organisation terroriste, lui apporter son soutien, même par un geste ou un signe lui faisant référence, devient un « acte terroriste ». Selon un porte-parole du ministère de l'Intérieur, la loi sera appliquée à toute personne qui «porte, met ou imprime le symbole de Rabia sur les réseaux sociaux». La peine pouvant aller jusqu'à cinq ans de prison, l'auteur d'un tel geste prend un risque certain. Très vite les internautes, d'Égypte notamment, retirent ceux de leurs portraits avec le geste de Rabia pour éviter de lourdes condamnations. Des sanctions, enfin, sont prises contre les sportifs auteurs du geste : des athlètes égyptiens sont ainsi déclarés interdits de Jeux olympiques, d'autres radiés définitivement des compétitions sportives. Dans les médias égyptiens et sur les réseaux sociaux, le geste de Rabia, appelé aussi le salut de Rabia, selon certains organes de presse ${ }^{25}$, devient cible d'ironie et de sarcasme, utilisé par les soutiens du président Sissi contre les Frères musulmans. Des photos circulent avec le geste de Rabia tenant un pistolet, ou représentant quatre doigts coupés avec du sang qui coule, pour accuser les Frères de violence et de terrorisme, ou bien encore transformant l'image en doigt d'honneur.

Le geste reste toutefois utilisé pour provoquer le pouvoir en place. À l'occasion de manifestations organisées de nuit, les graffitis représentant le geste et les slogans hostiles au régime recouvrent les murs de plusieurs villes égyptiennes, notamment Le Caire. Le nombre de blogs, de pages Facebook et Twitter portant le geste témoigne de la persistance des Frères musulmans et de leurs sympathisants à en faire un acte d'engagement et de combat. Hors des frontières, en juin 2015, une jeune étudiante défie encore le président Sissi, en visite en Allemagne, en lui adressant ce geste et en criant : «À bas le pouvoir militaire» lors d'un point de presse ${ }^{26}$.

L'analyse des origines, des interprétations du geste de Rabia et de ses usages dans la vie politique et médiatique en Égypte permet de prendre conscience de l'évolution de la situation dans ce pays après les événements qui ont suivi la chute du régime de Moubarak, la destitution de Morsi, puis l'arrivée au pouvoir du président Sissi.

Ce geste est devenu synonyme d'appartenance politique et idéologique, et de fait, il porte la marque d'une division profonde de la société entre deux parties : d'un côté, les partisans des Frères musulmans qui se présentent comme les défenseurs de la légitimité; de l'autre, ceux qui contestent leur vision politico-religieuse du monde et les décisions qu'ils ont impulsées. Enjeu

25. Voir par exemple: "The "Rabaa Salute" in 100 days: Politicians, athletes flash iconic hand-sign » (http://aa.com.tr/en/politics/the-rabaa-salute-in-10o-days-politicians-athletes-flash-iconichand-sign-timeline/202500) et «Four-finger salutes as Turks back Egypt protesters » (http:// www.reuters.com/article/2013/o8/19/us-egypt-turkey-idUSBRE97loPI20130819).

26. http://www.alaraby.co.uk/english/news/2015/6/5/celebrity-cheerleaders-and-youngstudent-tarnish-sisi-visit. 
symbolique d'un affrontement violent entre les deux camps, il est l'expression d'un mouvement de protestation inédit dans l'histoire du mouvement islamiste dans les pays arabes. Geste de mémoire, de défi et de résistance, investi de multiples significations religieuses et culturelles, il est devenu la marque emblématique du ralliement à une protestation politique dénonçant le refus du régime égyptien de mener à bien la démocratisation du pays. Sur l'autre bord, il est jugé suffisamment subversif par les autorités pour être réprimé et suffisamment menaçant par nombre de journalistes et d'internautes favorables au pouvoir pour être disqualifié. Les luttes symboliques qui se déroulent autour du geste soulignent en tout cas sa portée politique, dans cette période complexe du post-printemps arabe.

\section{Références}

ACLIMANDos Tewfik, 2011, "Splendeurs et misères du clientélisme », Égypte / Monde Arabe, $\mathrm{n}^{\circ} 7, \mathrm{p} .197-219$.

AlianAK Sonia, 2014, The Transition Towards Revolution and Reform: the Arab Spring Realized?, Edinbourg, Edinburgh University Press.

ANNESTAY Jean, 2009, Une femme soufie en Islam : Râbi‘a al-'Adawiyya, Paris, Entrelacs.

Arjomand Saïd Amir éd., 2015, The Arab Revolution of 2011. A Comparative Perspective, Albany, State University of New-York Press.

ARkoun Mohammed, 1986, L'Islam, morale et politique, Paris, Desclée de Brouwer / Unesco.

Austin Ralph W. Julius, MonteIL Vincent, Du PASquIER Roger, 1977, Le Monde arabe. Tradition et renouveau, Lausanne, Éditions des Trois Continents.

Azouzı Ammar, 2013, "La "Révolution du jasmin" en Tunisie et son slogan "Ben Ali dégage!" Un événement discursif», Les facettes de l'événement. Des formes aux signes, E. Ballardini, R. Pederzoli, S. Reboul-Touré, G. Tréguer-Felten éd., mediAzioni, n⿳015, http://mediazioni.sitlec.unibo.it.

BARbARY Caroline, AdiB Doss Maria, 2014, «Tamarrod («rébellion»). Une autre lecture de l'action politique dans le processus révolutionnaire égyptien », Confluences Méditerranée, n॰88, p. 155-169.

BENGHAL Jamal-Eddine, 2000, La vie de Rabi'a al-'Adawiyya. Une sainte musulmane du VIII' siècle, Paris, Iqra.

Berque Jacques, 1995, Le Coran. Essai de traduction, Paris, Albin Michel.

BoundiBA Abdelwahab, 1980, «Les Arabes et la couleur », Cahiers de la Méditerranée, n० 20-21, p. 63-77.

Boutaleb Assia 2014, "Les dynamiques de protestation et de la mobilisation en Égypte», Soulèvements et recompositions politiques dans le monde arabe, M. Camau, F. Vairel éd., Montréal, Presses universitaires de Montréal.

CARCENAC Claude-Brigitte, 2013, «Essai de recherche d'une typologie de la sainte mys- 
tique dans l'islam et le christianisme à partir de l'étude de cas de Rabia Adawiyya », Conserveries mémorielles [en ligne] : \#14 | 2013, URL: http://cm.revues.org/1606.

CARRÉ Olivier, Michaud Gérard, 1983, Les Frères musulmans, 1928-1982, Paris, Gallimard-Julliard.

Chebel Malek, 1993, Dictionnaire des symboles musulmans, rites, mystique et civilisation, Paris, Albin Michel.

Cole Juan, 2014, The New Arabs: How the Millennial Generation is Changing the Middle East, New York, Simon \& Schuster.

El ChAZLI Youssef, 2012, "Sur les sentiers de la révolution. Comment des Égyptiens "dépolitisés" sont-ils devenus révolutionnaires?", Revue française de science politique, vol.V, n62, p. 843-865.

FeUILLET Michel, 2004, Lexique des symboles chrétiens, Paris, PUF.

Gobillot Geneviève, Mystique musulmane. Parcours en compagnie d'un chercheur : Roger Deladrière, Actes du colloque du 9 mars 2001, Lyon, Université Jean-Moulin.

Guibal Claude, TANgI Salaun, 2011, L'Égypte de Tahrir. Anatomie d'une révolution, Paris, Seuil.

Khalifan Sherif, 2015, Egypt's Lost Spring: Causes and Consequences, Santa Barbara (California), Praeger.

LUIZARD Pierre-Jean, 1990, "Le soufisme égyptien contemporain», Égypte / Monde arabe, Première série, $\mathrm{n}^{\circ} 2$.

MASSIGNON Louis, 1987, Textes inédits présentés par J. Keryell, Paris, Nouvelle Cité.

Messiha Jean, Teulon Frédéric, 2013/2, «Égypte : d'une révolution à une autre?», Maghreb-Machrek, n²16, p. 129-142.

Mitchell Richard P., 1969, The Society of the Muslim Brothers, Oxford, Oxford University Press.

Rushdy Hatem éd., 2011, 18 Days in Tahrir: Stories from Egypt's Revolution, Londres, Havens Books.

SABEA Hanan, 2014, "I dreamed of being a people”: Egypt's revolution, the people, and critical imagination », The Political Aesthetics of Global Protest: The Arab Spring and Beyond, Édimbourg, Edinburgh University Press.

SMIth Margaret, 2010 [1928], Rābi'a the Mystic and her Fellow-Saints in Islām: Being the Life and Teachings of Rābi'a al-'Adawiyya Al-Qaysiyya of Basra. Together with Some Account of the Place of the Women Saints in Islām, Cambridge, Cambridge University Press. 